\title{
Controlling attosecond angular streaking with second harmonic radiation
}

\author{
T. J. Hammond, ${ }^{1, *}$ Kyung Taec Kim, ${ }^{1,2,3}$ Chunmei Zhang, ${ }^{1}$ D. M. Villeneuve, ${ }^{1}$ and P. B. Corkum ${ }^{1}$ \\ ${ }^{1}$ Joint Attosecond Science Laboratory, University of Ottawa and National Research Council of Canada, 100 Sussex Dr, \\ Ottawa K1N 5A2, Canada \\ ${ }^{2}$ Centre for Relativistic Laser Science, Institute for Basic Science (IBS), Gwangju 500-712, South Korea \\ ${ }^{3}$ Department of Physics and Photon Science, Gwangju Institute of Science and Technology (GIST), Gwangju 500-712, South Korea \\ *Corresponding author: thammond@uottawa.ca
}

Received January 27, 2015; revised March 16, 2015; accepted March 17, 2015; posted March 20, 2015 (Doc. ID 233219); published April 8, 2015

\begin{abstract}
High harmonic generation, which produces a coherent burst of radiation every half cycle of the driving field, has been combined with ultrafast wavefront rotation to create a series of spatially separated attosecond pulses, called the attosecond lighthouse. By adding a coherent second harmonic beam with polarization parallel to the fundamental, we decrease the generating frequency from twice per optical cycle to once. The increased temporal separation increases the pulse contrast. By scanning the carrier envelope phase, we see that the signal is $2 \pi$ periodic. (C) 2015 Optical Society of America

OCIS codes: (190.2620) Harmonic generation and mixing; (190.4160) Multiharmonic generation; (320.7110) Ultrafast nonlinear optics.

http://dx.doi.org/10.1364/OL.40.001768
\end{abstract}

The attosecond lighthouse, driven by a few cycle pulse, can generate several high flux, mutually coherent, spatially separated beamlets, each with a bandwidth extending up to a cutoff energy that is dependent on the instantaneous driving field intensity [1,2]. The spatially separated, sequentially generated beamlets map the temporal dependence of high harmonic generation to space [3]. For a noble gas, the attosecond bursts are produced twice per optical cycle due to symmetry. We will show that coherently adding the driving field and its second harmonic allows for sub-cycle manipulatation of the generation process. Specifically, a sufficiently strong second harmonic breaks the symmetry and controls the ionization rate, the first step in high harmonic generation [4,5].

Symmetry breaking may also be attained in the generating medium by using nonsymmetric, oriented polar molecules [ $\underline{6}-\underline{8}]$. The relative amplitudes of the separated beamlets from the attosecond lighthouse can directly measure the change in ionization from oriented polar molecules. Understanding the second harmonic influence on the ionization process of an atomic gas is an initial step in understanding high harmonic spectroscopy from oriented, polar molecules $[9,10]$.

Modifying the relative phase between the second harmonic and the driving field modulates the ionization rate, promoting or inhibiting electron tunnelling every half cycle. If the second harmonic intensity is large enough, ionization is confined to a single time window per optical cycle and, consequently, attosecond bursts are only generated once per cycle $[11,12]$. Increased temporal separation leads to an increased spatial separation of the generated beamlets, improving the pulse contrast of the attosecond lighthouse $[13,14]$.

A schematic of the experimental setup is shown in Fig. 1. We used a carrier envelope phase (CEP) stabilized $1 \mathrm{kHz}$ Ti:sapphire laser generating $1.5 \mathrm{~mJ}$ pulses at $25 \mathrm{fs}$ centered at $790 \mathrm{~nm}$. With a differentially pumped hollow core neon-filled fiber operated at 2 bar and dispersion compensation, we attained $300 \mu \mathrm{J}$, sub-5-fs pulses centered at $760 \mathrm{~nm}$ [3].

The second harmonic was generated by a $200 \mu \mathrm{m}$ thick $\beta$-barium borate (BBO) crystal phase matched to produce a second harmonic centered at $410 \mathrm{~nm}, 1.85$ of the fundamental carrier frequency, to maximize the intensity after reflection from protected Ag mirrors. The second harmonic was split from the fundamental beam with a harmonic separator. In this configuration, the second harmonic intensity was approximately $3 \times 10^{12} \mathrm{~W} / \mathrm{cm}^{2}$ (a relative electric field $E(2 \omega) / E(\omega)=8 \%$ ). The estimate was based on a measured pulse energy of $3 \mu \mathrm{J}$, spot size of $35 \mu \mathrm{m}$, and an estimated pulse duration of $25 \mathrm{fs}$. We controlled the second harmonic delay with a closed-loop piezo stage. The second harmonic polarization was rotated with a $\lambda / 2$ wave plate at $400 \mathrm{~nm}$, and was recombined colinearly with the driving field with a harmonic combiner. The beam recombination took place after the wedges because their spatial and temporal dispersion

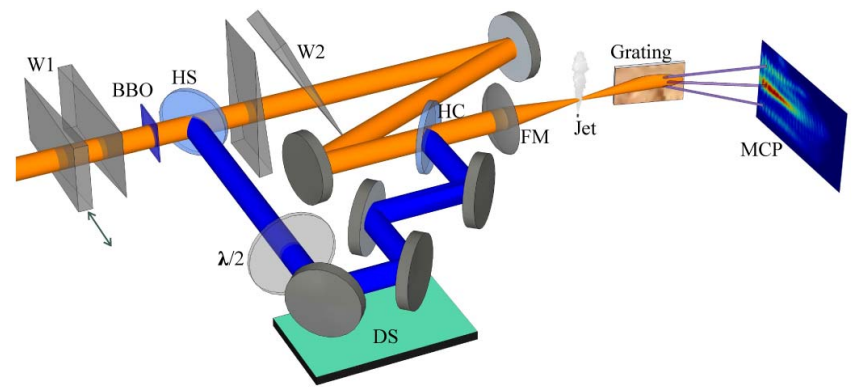

Fig. 1. Schematic of the attosecond lighthouse with the second harmonic. A pair of wedges W1 scanned the CEP; A $200 \mu \mathrm{m}$ thick $\beta$-BBO generated the second harmonic, phase matched at $410 \mathrm{~nm}$; harmonic separator (HS); misaligned wedges used to generate the spatial chirp W2; $\lambda / 2$ half waveplate; computer controlled piezo delay stage DS; harmonic combiner (HC); focusing silver mirror $f=300 \mathrm{~mm} \mathrm{~F}$; 4 bar backing neon pulsed gas jet; grating $1200 \mathrm{l} / \mathrm{mm}$; micro-channel plate MCP to image. 
limited the second harmonic intensity. The consequence of using the second harmonic at not exactly twice the fundamental frequency is that the phase of the second harmonic is not optimized to enhance or quench all the beamlets simultaneously.

We imposed a spatial chirp on the fundamental beam using a pair of misaligned wedges (relative angle $24^{\circ}, \mathrm{BK} 7$ $8^{\circ}$ apex angle). The peak intensity of the fundamental field with the ultrafast wavefront rotation is estimated to be $4.5 \times 10^{14} \mathrm{~W} / \mathrm{cm}^{2}$ based on spot size $(40 \mu \mathrm{m} \times$ $55 \mu \mathrm{m})$, pulse power, and duration; and we confirmed the intensity via the maximum cutoff energy.

Perfectly matched wavefronts of the fundamental and its second harmonic could allow for increased control from the second harmonic. In this experiment, we do not impose spatial chirp on the second harmonic and, although the second harmonic wavefront does not perfectly match the entire rotating wavefront of the fundamental driving field at the focus, the $3 \mathrm{mrad}$ of rotation per optical cycle for a few cycle pulse limits the wavefront mismatch between the fundamental and its second harmonic. As such, it is straightforward to observe a strong effect from the second harmonic on the generated beamlets.

The gas jet had a $250 \mu \mathrm{m}$ diameter aperture and was backed with 4 bar neon. The jet was placed $0.5 \mathrm{~mm}$ before the focus (found by measuring the position of the peak ionization rate) to minimize the spatial divergence of the short trajectory creating the attosecond pulses.

The generated pulses passed through a $0.4 \mathrm{~mm}$ slit, were spectrally dispersed by a $1200 \mathrm{l} / \mathrm{mm}$ grating, and were recorded on an MCP located $1 \mathrm{~m}$ from generating source. The spectrum was imaged with a CCD camera with a $1 \mathrm{~s}$ acquisition time. The horizontal axis records the spectrum and the vertical axis records the spatial distribution.

In Fig. 2, we compare the attosecond lighthouse spectra with and without a strong second harmonic. The left column (a)-(c) is the near-field dipole moment $|d(t, y)|^{2}$ calculated by the strong field approximation (SFA) from the measured values of the driving field (pulse energy, duration, and spot size), with a temporal filter removing the long trajectories. The dashed arrows from (a) to (d) connect the calculated near-field attosecond pulses to the associated measured beamlets, each propagating at a slightly different angle. The middle column (d)-(f) shows the experimentally measured spatial-spectral distributions, while the right column (g)-(i) is the spatial distribution summed over the energy range $30-80 \mathrm{eV}$. The calculated attosecond pulses in Fig. 2(a) have been numbered -3 to 3 and mapped to their associated measured beamlets in Fig. 2(g); the beamlets are discussed by their respective numbers.

The effect of only the fundamental with ultrafast wavefront rotation without the second harmonic present is shown in the top row of Figs. 2(a), 2(d), and 2(g). The measured lighthouse spectrum is shown in Fig. 2 (d), where the CEP is set to $\pi / 2$. The spectral modulation on each beamlet is because of a slight spatial overlap with adjacent beamlets in the far field. The modulation is a better measure of the spatial overlap than the total spectral amplitude because the latter is influenced by the CEP fluctuations, approximately $400 \mathrm{mrad}$ single shot [3]
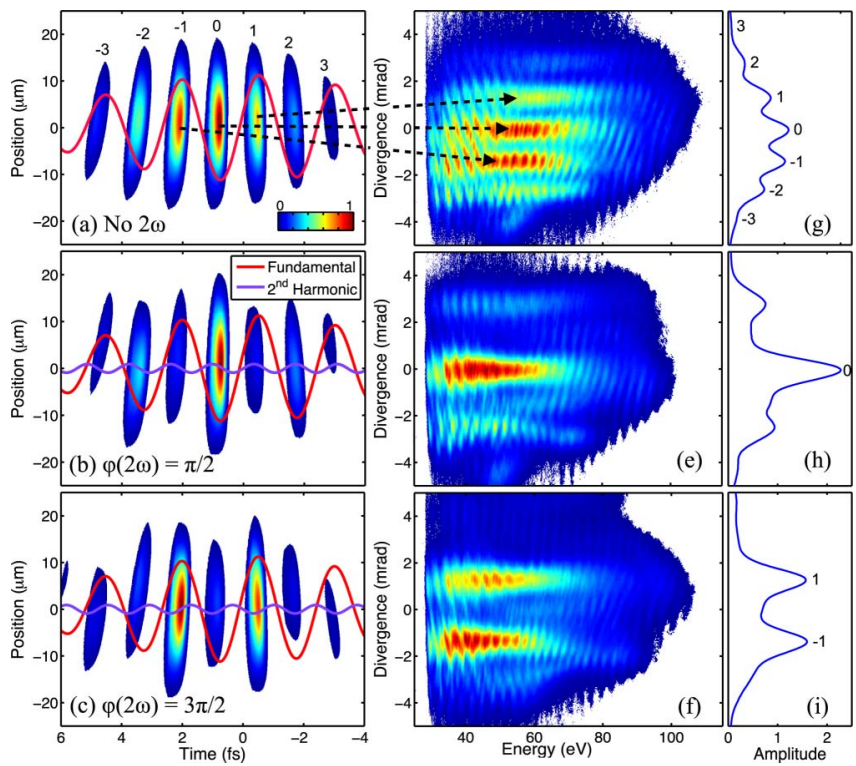

Fig. 2. Left column (a)-(c): near-field SFA calculation matching experimental conditions, with fundamental field (red) and second harmonic (blue). Middle column (d)-(f): measured farfield spatial-spectral distribution of the attosecond lighthouse. Right column (g)-(i): spatial distributions summed over 30 $80 \mathrm{eV}$. Each color image (a)-(f) is normalized to unity, where the total amplitudes are given in the angular totals (g)-(i). Top row (a), (d) and (g): without the second harmonic the emission occurs every half cycle, with CEP set to $\pi / 2$; middle row (b), (e) and (h): with the second harmonic phase set to $\phi(2 \omega)=\pi / 2$, the on-axis emission amplitude and pulse contrast are increased; bottom row (c), (f) and (i): $\phi(2 \omega)=3 \pi / 2$, the second harmonic decreased the central pulse and increased the satellite pulses, generating multiple, well-isolated, broadband attosecond pulses.

The middle row, Figs. 2(b), 2(e), and 2(h), shows the second harmonic beam added to the fundamental, where the phase favors the central, 0 beamlet. In Fig. 2(h), we see that the 0 beamlet is 2.3 times stronger than without the second harmonic. Simultaneously, the figure shows that the \pm 1 beamlets have their relative amplitudes reduced by 1.5 and 1.2 times, respectively. The relative enhancement (quenching) of the beamlet amplitude agrees with the change in ionization rate because of the second harmonic calculated from the tunneling rate [15]. The reduced spectral modulation on the central beamlet improves the contrast ratio by a factor of 2.5 , where the modulation amplitude implies a contrast ratio of greater than 3000 [12]. The improved contrast is less than expected from the total amplitude factor because the spectral amplitude is not uniformly changed by the presence of the second harmonic. Higher second harmonic intensity would further inhibit satellite pulse generation and increase the attosecond pulse contrast [5]

Delaying the second harmonic by $\pi$ leads to the bottom row, Figs. 2(c), 2(f) and 2(i). Here, the 0 beamlet is suppressed by a factor of $1 . \overline{4}$ while the \pm satellite beamlets are promoted by 2.5 and 2.1, respectively. Each of these two promoted, high-flux pulses has a bandwidth sufficient to support a transform limited pulse duration of $\sim 40$ as. The attenuation of the 0 beamlet could allow for easier alignment of the \pm 1 beamlets when used as 

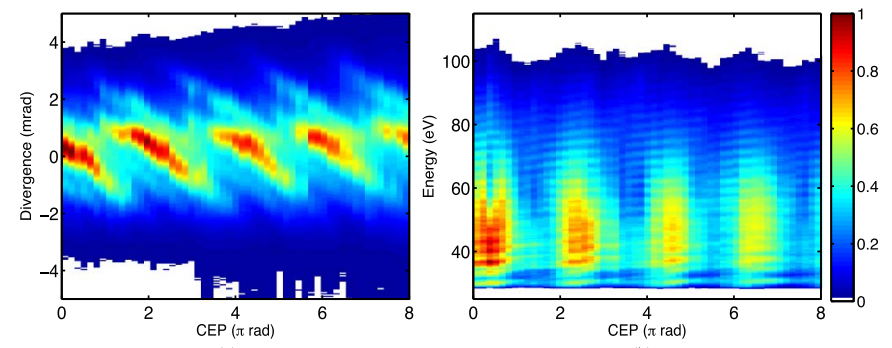

(a)

Fig. 3. Spatial distribution of the attosecond lighthouse while scanning the CEP with the second harmonic present. (a) The direction of the beamlets is CEP dependent, where the signal is now modulo $2 \pi$ of the driving field because of the second harmonic. The spectrum is summed over $30-80 \mathrm{eV}$. (b) The spectral amplitude of the on-axis pulses generated over a $1 \mathrm{mrad}$ divergence. The on-axis beamlet amplitude is now strongly dependent on the CEP.

a source for attosecond pump-attosecond probe experiments [16-18].

We also scanned the CEP using a pair of aligned wedges. This scan occurs before the BBO produces the second harmonic, thus the phase of the second harmonic, set to $\pi / 2$ when the CEP is $\pi / 2$, follows that of the fundamental. The spatially resolved spectrum, summed over 30-80 eV, is shown in Fig. 3(a) as a function of the CEP. Because the driving field phase is determined by the CEP, in the presence of ultrafast wavefront rotation scanning the CEP allows for a continuous rotation of the wavefront, and consequently scanning the CEP continuously changes the direction of the generated beamlets. With the second harmonic present, the signal is modulo $2 \pi$ [2]. Thus, the presence of the second harmonic removes the $\pi$ ambiguity of the driving field phase $[\underline{19}, 20]$. The consequence of using the attosecond lighthouse with the second harmonic as a source for attosecond pulses is shown in Fig. 3(b). An on-axis aperture of $1 \mathrm{mrad}$ diameter attenuates the attosecond pulses generated when the CEP has changed by $\pi$. With a stronger second harmonic and an appropriate spatial filter, it may be possible to completely suppress attosecond pulses generated with undesired CEP [21].

In conclusion, we have shown that a parallel polarized second harmonic beam can control the beamlets generated by the attosecond lighthouse. With a second harmonic field amplitude of only $8 \%$ of the fundamental, we can increase the temporal and spatial separation of the beamlets. Even with such a weak control beam, the contrast between a prepulse and the main pulse is comparable to other attosecond pulse generation methods [22]. In addition, the increased separation lessens the requirements for ultrafast wavefront rotation. To achieve similar beamlet spatial separation and isolation as the initially sub-5-fs generating pulse case, the pulse duration can be increased to $\sim 10$ fs. [23].

Further increasing the second harmonic intensity increases its influence over the spectrum and phase of the attosecond pulses. Looking forward, using the second harmonic to shape the attosecond pulse may be an efficient method to generate attosecond pulses reaching a single atomic unit of time ( $\sim 24$ as).

We acknowledge support from the DARPA Pulse program through a grant from AMRDEC and from the AFOSR. We also acknolwledge continuing support from Canada's NRC, NSERC and CFI. We are grateful for the helpful discussions with Andrei Naumov and technical assistance from Bert Avery and David Crane.

\section{References}

1. H. Vincenti and F. Quéré, Phys. Rev. Lett. 108, 113904 (2012).

2. J. A. Wheeler, A. Borot, S. Monchocé, H. Vincenti, A. Ricci, A. Malvache, R. Lopez-Martens, and F. Quéré, Nat. Photonics 6, 829 (2012).

3. K. T. Kim, C. Zhang, T. Ruchon, J.-F. Hergott, T. Auguste, D. M. Villeneuve, P. B. Corkum, and F. Quéré, Nat. Photonics 7, 651 (2013).

4. N. Dudovich, O. Smirnova, J. Levesque, Y. Mairesse, M. Y. Ivanov, D. M. Villeneuve, and P. B. Corkum, Nat. Phys. 2, 781 (2006).

5. M. Fieß, B. Horvath, T. Wittmann, W. Helml, Y. Cheng, B. Zeng, Z. Xu, A. Scrinzi, J. Gagnon, F. Krausz, and R. Kienberger, New J. Phys. 13, 033031 (2011).

6. A. Etches and L. B. Madsen, J. Phys. B 43, 155602 (2010).

7. E. Frumker, C. T. Hebeisen, N. Kajumba, J. B. Bertrand, H. J. Wörner, M. Spanner, D. M. Villeneuve, A. Naumov, and P. B. Corkum, Phys. Rev. Lett. 109, 113901 (2012).

8. P. M. Kraus, A. Rupenyan, and H. J. Wörner, Phys. Rev. Lett. 109, 233903 (2012).

9. E. Frumker, N. Kajumba, J. B. Bertrand, H. J. Wörner, C. T. Hebeisen, P. Hockett, M. Spanner, S. Patchkovskii, G. G. Paulus, D. M. Villeneuve, A. Naumov, and P. B. Corkum, Phys. Rev. Lett. 109, 233904 (2012).

10. P. M. Kraus, D. Baykusheva, and H. J. Wörner, Phys. Rev. Lett. 113, 023001 (2014).

11. J. Mauritsson, P. Johnsson, E. Gustafsson, A. L'Hullier, K. J. Schafer, and M. B. Gaarde, Phys. Rev. Lett. 97, 013001 (2006).

12. H. Mashiko, S. Gilbertson, C. Li, S. D. Khan, M. M. Shakya, E. Moon, and Z. Chang, Phys. Rev. Lett. 100, 103906 (2008).

13. T. Pfeifer, L. Gallmann, M. J. Abel, D. M. Neumark, and S. R. Leone, Opt. Lett. 31, 975 (2006).

14. Y. Oishi, M. Kaku, A. Suda, F. Kannari, and K. Midorikawa, Opt. Express 14, 7230 (2006).

15. G. L. Yudin and M. Y. Ivanov, Phys. Rev. A 64, 013409 (2001)

16. E. Goulielmakis, Z.-H. Loh, A. Wirth, R. Santra, N. Rohringer, V. S. Yakovlev, S. Zherebtsov, T. Pfeifer, A. M. Azzeer, M. F. Kling, S. R. Leone, and F. Krausz, Nature 466, 739 (2010).

17. J. Mauritsson, T. Remetter, M. Swoboda, K. Klünder, A. L'Huillier, K. J. Schafer, O. Ghafur, F. Kelkensberg, W. Siu, P. Johnsson, M. J. J. Vrakking, I. Znakovskaya, T. Uphues, S. Zherebtsov, M. F. Kling, F. Lépine, E. Benedetti, F. Ferrari, G. Sansone, and M. Nisoli, Phys. Rev. Lett. 105, 053001 (2010).

18. C. Lefebvre, T. T. Nguyen-Dang, F. Dion, M. J. J. Vrakking, V. N. Serov, and O. Atabek, Phys. Rev. A 88, 053416 (2013).

19. G. G. Paulus, F. Grasbon, H. Walther, P. Villoresi, M. Nisoli, S. Stagira, E. Priori, and S. De Silvestri, Nature 414, 182 (2001).

20. T. Wittmann, B. Horvath, W. Helml, M. G. Schätzel, X. Gu, A. L. Cavalieri, G. G. Paulus, and R. Kienberger, Nature Phys. 5, 357 (2009).

21. S. Gilbertson, S. D. Khan, Y. Wu, M. Chini, and Z. Chang, Phys. Rev. Lett. 105, 093902 (2010).

22. C. Altucci, J. W. G. Tisch, and R. Velotta, J. Mod. Opt. 58, 1585 (2011).

23. Z. Chang, Phys. Rev. A 76, 051403 (2007). 